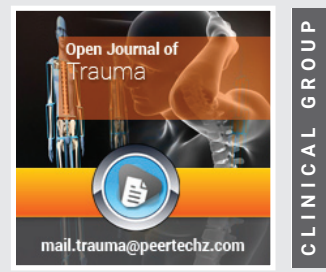

\title{
Trauma Resuscitation Time at A Tertiary Care Hospital in Oman: A Retrospective Observational Study
}

Received: 28 May, 2020

Accepted: 11 June, 2020

Published: 12 June, 2020

*Corresponding author: Salim Al Rawahi, Division of Orthopaedic Surgery, McGill University, Montreal, Qc, Canada, Tel: (+1) 438680 5361; E-mail: salrawahi99@gmail.com

Keywords: Resuscitation time; Trauma; Surgery https://www.peertechz.com

Check for updates

\author{
${ }^{1}$ Department of Surgery, Sultan Qaboos University Hospital, Muscat, Oman \\ ${ }^{2}$ Division of Orthopaedic Surgery, McGill University, Montreal, Qc, Canada
}

Maysa Al-Maawali ${ }^{1}$, Salim Al Rawahi ${ }^{2 \star}$ and Hani Al-Qadhi ${ }^{1}$

\section{Abstract}

Introduction: Trauma is an important worldwide public-health problem. This study aims to evaluate the median trauma resuscitation time (TRT) at Sultan Qaboos University Hospital (SQUH), Oman, and to investigate the reasons for delay in trauma resuscitation. Documenting TRT can be utilized to assess areas of improvement for trauma team performance, ensuring a high-quality resuscitation management that improves trauma patients' survivability.

Methods: This is a retrospective observational study. It included all trauma patients attended the Emergency Department (ED) and required trauma team resuscitation in the period between June 2016 and June 2017. Electronic Health Records (EHR) were accessed to retrieve data of 295 patients included. The TRT was obtained by calculating the difference between the time of the patient's arrival to the trauma bay and the time of the patient's disposition. Patients' demographics and clinical data such as GCS, trauma mechanism, trauma type, resuscitation procedures and outcomes were evaluated.

Results: A total of 270 patients were included in the final results; 25 patients were excluded for missing data. The median TRT was found to be 25 minutes (IQR= 17); the mean age was $26.9 \pm 13.9$ years; $85.6 \%$ patients were male; the mechanisms of injury were: motor vehicle collision (MVC) (45.9\%), pedestrian struck (25.2\%), fall $(17.4 \%)$, stab injury (5.9\%), crush injury (4.4\%), burn $(0.7 \%)$ and assault $(0.4 \%) ; 67(52.3 \%)$ patients required intubation, 19 (14.8\%) patients required chest tube insertion, 2 (1.5\%) patients required a central line was insertion, 13 (4.8\%) patients required focused assessment with sonography in trauma (FAST) and 27 (10\%) patients required cast application for upper and lower extremity fractures; $202(74.8 \%)$ patients were admitted directly from the emergency department, 14 (5.2\%) patients required emergent surgery, 34 (12.5\%) patients were discharged home, 4 (1.5\%) patients were referred to a different hospital and 16 (5.9\%) patients died. Of those who died, 14 patients died during the resuscitation.

Conclusion: We concluded from this study that patients with stab injury required more resuscitation time and the delay in resuscitation was observed among those who required FAST examination and intubation. Advanced Trauma Life Support training (ATLS), simulation-based training, and video review have all improved patient outcome and trauma team performance. Further prospective studies are warranted to address the limitations.

\section{Introduction}

Trauma is a worldwide major cause of death and disability. It accounts for $9 \%$ of global mortality annually and $10 \%$ of morbidity and mortality in Oman, particularly those related to road safety [1]. In fact, it continues to be a worldwide burden on all societies and may out-pace infectious diseases as a leading cause of worldwide mortality [2]. Since the initial hour after an injury is the most crucial time for trauma patients, early resuscitation is of vital importance. Trauma Resuscitation is defined as "assessment and stabilization of vital functions and prioritization of therapeutic and diagnostic interventions for the severely injured patient arriving in the Emergency Department [3]." Time is considered an essential determinant in the initial care of trauma patients, as rapid effective trauma resuscitation decreases patient morbidity and mortality. Trauma resuscitation time (TRT) is defined as "the time between the actual arrival of the patient into the emergency department trauma bay and the final disposition" [4]. This initial period is directly related to the patients' outcomes and delays might result in preventable deaths [5]. Different retrospective studies have reported different trauma resuscitation time with a median 
time ranging from fifteen minutes to a hundred and fifty-six minutes [3-7]. To our knowledge, this is the first study to investigate trauma resuscitation time in Oman. We, therefore, aim to evaluate the trauma resuscitation time among trauma patients who attended Sultan Qaboos University Hospital during the period from June 2016 to June 2017. In addition, we aim to identify the causes of delay in trauma resuscitation in order to improve trauma team performance and the quality of trauma resuscitation.

\section{Materials and methods}

\section{Study design and population}

This is a retrospective observational study that was conducted at the Sultan Qaboos University Hospital (SQUH), Muscat, Oman. It included all trauma patients who attended the Emergency Department (ED) and required trauma resuscitation by the trauma team in the period between June 2016 and June 2017. We excluded patients with minor traumatic injuries who did not require trauma team assessment, patients who were managed at and referred from different hospitals, regular trauma consults, and patients who were initially managed by emergency physicians. The study was approved by the Research and Ethics Committee in the College of Medicine and Health Sciences, Sultan Qaboos University.

\section{Data collection}

Electronic health records (HER) via (Trakcare ${ }^{\circledR}$ system) were reviewed to extract data of all eligible patients included in the study. These data include: patients' demographics, trauma type, mechanism of injury, Glasgow Coma Scale (GCS), resuscitation time, resuscitation procedures and patients' outcomes. The emergency trauma bay entry-to-exit time was considered as the trauma resuscitation time (TRT). The exit time represents the end of the resuscitation time, which includes transferring the patient to the radiology department, operating theater (OR), admission, discharge, and death.

\section{Data analysis}

Statistical Package for the Social Sciences (SPSS, version 23.0) was used for statistical analysis. Descriptive analyses were applied to evaluate the median trauma resuscitation time, mean age, frequency of trauma mechanisms, resuscitation procedures, and outcomes. Chi-square and Fisher's exact tests were used to evaluate the statistical significance of differences among categorical data. Mann-Whitney U test and KruskalWallis $\mathrm{H}$ test were used to compare the resuscitation time between the groups of the categorical data. A p-value of less than 0.05 was considered significant.

\section{Results}

A total of 295 patients met the inclusion criteria. Of those, 25 patients were excluded because of missing data in their records. A total of 270 patients were included in the final results. The age of the study population ranged from 1 to 86 years, with a mean of $26.9 \pm 13.9$ years. Male predominance accounts for $85.6 \%$ (Table 1 ).
Table 1: Demographic data.

\begin{tabular}{|c|c|c|c|c|c|c|}
\hline \multirow{2}{*}{ Age } & \multicolumn{2}{|c|}{ Male $(n=231)$} & \multicolumn{2}{|c|}{ Female $(n=39)$} & \multicolumn{2}{|c|}{ Total $(\mathrm{N}=270)$} \\
\hline & $n$ & $\%$ & $n$ & $\%$ & $n$ & $\%$ \\
\hline$\leq 12$ years & 26 & 65.0 & 14 & 35.0 & 40 & 14.8 \\
\hline $13-59$ years & 201 & 89.7 & 23 & 10.3 & 224 & 82.9 \\
\hline 60 years & 4 & 66.7 & 2 & 33.3 & 6 & 2.2 \\
\hline
\end{tabular}

Two-hundred and fifty-four (94.1\%) patients experienced blunt traumatic injuries and $16(5.9 \%)$ patients were victims of penetrating injuries. The most frequent mechanism of injury was motor vehicle collision (MVC) (45.9\%); followed by pedestrian struck (25.2\%); fall (17.4\%); stab injury $(5.9 \%)$; crush injury (4.4\%); burn (0.7\%) and assault (0.4\%). A hundred and twenty-eight $(47.4 \%)$ patients required one or more of the resuscitation procedures: $67(52.3 \%)$ patients required intubation, $19(14.8 \%)$ patients required chest tube insertion, and $2(1.5 \%)$ patients required a central line insertion. In addition, $13(4.8 \%)$ patients required focused assessment with sonography in trauma (FAST) and $27(10 \%)$ patients required cast application for upper and lower extremity fractures (Figure 1).

Two-hundred and two (74.8\%) patients were admitted directly from the emergency department, 14 (5.2\%) patients required emergent surgery, $34(12.5 \%)$ patients were discharged home, $4(1.5 \%)$ patients were referred to a different hospital and $16(5.9 \%)$ patients died. Of those who died, 14 patients died during the resuscitation. The median trauma resuscitation time was found to be 25 minutes (interquartile range $(I Q R)=17$ ). There was no statistical significance between GCS, age, gender, mechanism of injury and trauma resuscitation time. However, there was a statistically significant correlation between the trauma resuscitation time and death $(p$-value $=0.004)$. The trauma resuscitation time and patients' characteristics are described in Table 2.

Binary logistic regression analysis demonstrated that GCS $\leq 8$ and intubation were significantly associated with death $(p$-value $=0.0001$ and 0.024 , respectively) whereas trauma resuscitation time and mechanism of injury are independent causes of mortality (Table 3).

\section{Discussion}

In our study, a higher number of trauma visits was observed among male adults (85.6\%), a finding that is consistent with different studies $[7,9,10]$. The median trauma resuscitation time was 25 minutes, making it comparable with internationally recognized trauma centers [2-5]. However, a

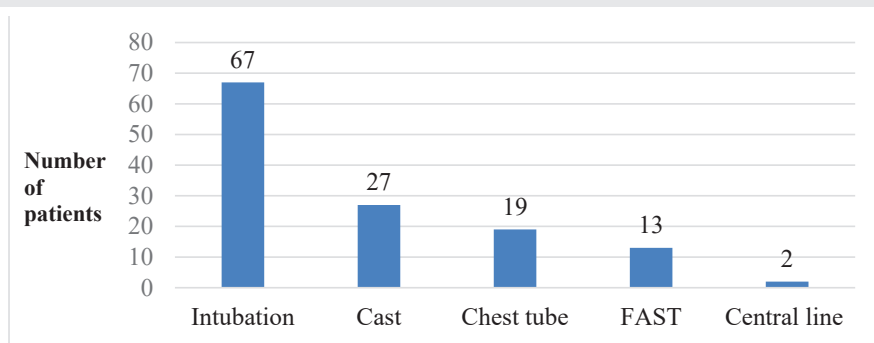

Figure 1: Distribution of procedures among trauma patients.

Citation: Al-Maawali M, Al Rawahi S, Al-Qadhi H (2020) Trauma Resuscitation Time at A Tertiary Care Hospital in Oman: A Retrospective Observational Study. Open 
Table 2: Trauma resuscitation time and patients' variables.

Characteristics

\section{Age}

$\leq 12$

$13-59$

$\geq 60$

Gender

Male

Female

GCS

13-15

$9-12$

$\leq 8$

MVC

Fall

Pedestrian

Stab

Crush

Assault

Burn

Procedures

Intubation

Chest tube

Cast

FAST

Central line insertion

Outcome

Admission

Discharge

Death

Referral
Mechanism of trauma
Resuscitation time in minutes Median

p-value*

24.5

25.0

22.0

25.0

30.0

25.0

24.0

22.5

23.0

28.0

24.0

31.5

23.5

17.0

96.0

29.0

22.0

21.0

29.0

17.0

25.5

23.5

19.0

57.0

0.54

0.152

0.26

0.19

0.06

0.27

0.03

0.68

0.32

0.25

0.01

0.28

0.06

0.04

0.11

0.92

0.19

0.004

0.27
GCS: Glasgow Coma Scale; MVC: Motor Vehicle Collision; FAST: Focused Assessment with Sonography for Trauma

Table 3: Binary logistic regression analysis to determine the predictors of death.

\begin{tabular}{|c|c|c|c|c|c|}
\hline \multirow{2}{*}{ Variable } & \multirow{2}{*}{ B } & \multirow{2}{*}{ Sig. } & \multirow{2}{*}{ Odds Ratio (OR) } & \multicolumn{2}{|c|}{ 95\% Cl for OR } \\
\hline & & & & Lower & Upper \\
\hline \multicolumn{6}{|l|}{ Trauma mechanism } \\
\hline \multicolumn{6}{|l|}{ MVC (reference) } \\
\hline Fall from height & .775 & .547 & 2.171 & .174 & 27.001 \\
\hline Stabbing & -15.485 & .999 & .000 & & \\
\hline Pedestrian & .857 & .289 & 2.356 & .483 & 11.499 \\
\hline Crush injury & 1.880 & .177 & 6.555 & .428 & 100.311 \\
\hline Burn & -12.595 & 1.000 & .000 & & \\
\hline Others & -19.924 & 1.000 & .000 & & \\
\hline \multicolumn{6}{|l|}{ Glasgow coma scale } \\
\hline \multicolumn{6}{|l|}{ 13-15 (reference) } \\
\hline 9-12 & -16.739 & .999 & .000 & & \\
\hline$\leq 8$ & 4.990 & $0.0001^{*}$ & 146.918 & 16.241 & 1329.011 \\
\hline \multicolumn{6}{|l|}{ Intubation } \\
\hline \multicolumn{6}{|l|}{ No (reference) } \\
\hline Yes & -2.192 & $0.024^{*}$ & .112 & .017 & .745 \\
\hline Resuscitation time & -.084 & .072 & .919 & .838 & 1.008 \\
\hline
\end{tabular}

minimal resuscitation time recorded was 15 minutes in a study investigating the variation in trauma resuscitation and its effect on patient outcome [7]. This study demonstrated that the shorter yet efficient resuscitation is the key for a better patient's outcome, regardless of type of patient dealt with, seniority of the team leader or the number of personnel in the trauma team.

The most frequent mechanism of injury was MVC (45.9\%) followed by pedestrian struck (25.2\%). Although road traffic accidents (RTA) show a declining trend in Oman, this is an expected result [11]. Patients with stab injuries significantly required more resuscitation, 31.5 minutes $(P=0.038)$. These patients required additional diagnostic and imaging tests to be done in the trauma bay which could explain the lengthy resuscitation time. Sixty-seven $(52.3 \%)$ patients required intubation while few patients had FAST and central line insertion. Intubation was indicated for patients with GCS $\leq 8$, airway obstruction, respiratory failure and to manage agitated patients. Thirty-nine $(58.2 \%)$ of the intubated patients had GCS of less than 8 ( $p$-value $<0.0001)$. Patients who required intubation and FAST demonstrated a significantly longer resuscitation time, 29 minutes ( $p$-value $=0.01$ and 0.04 , respectively). This raises the importance of having well-trained clinicians, who can invariably perform intubation and FAST examination to aid rapid decision making. There is a major national and international variety in trauma team composition. However, and based on the aforementioned results, all trauma team members should be well-trained on performing diagnostic and life-saving interventions, particularly FAST and intubation.

The underlying cause of death in this study could be attributed to the severity of the injuries as these patients required less resuscitation time than the average (19 minutes vs. 25 minutes) and died during the resuscitation.

Patients with severe burn and hand injuries were transferred to a different hospital that is equipped with plastic surgery and burn unit; the resuscitation time of these patients was the longest (57 minutes) yet statistically insignificant ( $p$-value= 0.27 ). We believe that more time was needed to arrange for the transfer than resuscitating the patients. The exit time from the trauma bay was a significant source of delay and therefore, prolonging the trauma resuscitation time meant to be studied. The time between last intervention in the trauma bay and disposal is the greatest contributor to total time spent in the emergency department [12].

The current study has a number of limitations: it's a retrospective observational study, has a small sample size, and it did not address the accuracy of diagnosis, injury severity score (ISS), length of each procedure performed in the trauma bay, and effectiveness of resuscitation.

\section{Conclusion}

We concluded from this study that patients with stab injuries required more resuscitation time and the delay in resuscitation was observed among those who required FAST examination and intubation. A delay in trauma resuscitation is crucial and performing resuscitation procedure competently is of paramount importance. Advanced Trauma Life Support training (ATLS), simulation-based training, and video review have all improved patient outcome and trauma team performance. Further prospective studies are warranted to address the limitations.

\section{References}

1. Review of the injury surveillance system. World Health Organization Regional Office for the Eastern Mediterranean website, oman-news, Oman. Link: https://bit.ly/3hgZ5YT 
2. Krug EG, Sharma GK, Lozano R (2000) The global burden of injuries. Am J Public Health 90: 523-526. Link: https://bit.ly/3f7zqzU

3. Bonjour TJ, Charny G, Thaxton RE (2016) Trauma Resuscitation Evaluation Times and Correlating Human Patient Simulation Training Differences-What is the Standard. Mil Med 181: e1630-e1636. Link: https://bit.ly/3hjhkgc

4. Van Olden G, Van Vugt AB, Biert J, Goris JA (2003) Trauma resuscitation time. Injury 34: 191-195. Link: https://bit.ly/3cVX80y

5. Lowe D, Pope R, Hedges J (1990) Time-management study of trauma resuscitation. Am J Surg 159: 457-461. Link: https://bit.ly/37hDNpw

6. Hoyt DP, Shackford SR, Fridland PH, Mackersie RC, Hansbrough JF, et al (1988) Video Recording Trauma Resuscitations: An Effective Teaching Technique. J Trauma 28: 435-440. Link: https://bit.ly/2UvNCL6

7. Driscoll PA, Vincent CA (1992) Variation in trauma resuscitation and its effect on patient outcome. Injury 23: 111-115. Link: https://bit.ly/2Ynve8D
8. Spanjersberg WR, Bergs EA, Mushkudiani N, Klimek M, Schipper IB (2009) Protocol compliance and time management in blunt trauma resuscitation. Emerg Med J 26: 23-27. Link: https://bit.ly/3hhI4xL

9. Al-Balushi H, Al-Kalbani A, Al-Khwaldi T, Al-Suqri S, Al-Maniri A, et al. (2012) Injuries Presented at a Primary Care Setting in Oman. OMJ 27: 486-490. Link: https://bit.ly/2YrXiHV

10. Al-Kashmiri A (2018) Trauma Care in Oman: Where do we stand and where should we be heading. SQUMJ 17: 386. Link: https://bit.ly/30ya3U2

11. Mazharul I, Al Hadhrami A (2012) Increased Motorization and Road Traffic Accidents in Oman. JETEMS 3: 907-914. Link: https://bit.ly/2zspbao

12. Tan TX, Quek NX, Koh ZX, Nadkarn N, Singaram K, et al. (2016) The Effect of Availability of Manpower on Trauma Resuscitation Times in a Tertiary Academic Hospital. PLoS One 11: 0154595. Link: https://bit.ly/30tVBwc

Discover a bigger Impact and Visibility of your article publication with Peertechz Publications

\section{Highlights}

- Signatory publisher of ORCID

* Signatory Publisher of DORA (San Francisco Declaration on Research Assessment)

* Articles archived in worlds' renowned service providers such as Portico, CNKI, AGRIS, TDNet, Base (Bielefeld University Library), CrossRef, Scilit, J-Gate etc.

* Journals indexed in ICMJE, SHERPA/ROMEO, Google Scholar etc.

* OAI-PMH (Open Archives Initiative Protocol for Metadata Harvesting)

* Dedicated Editorial Board for every journal

* Accurate and rapid peer-review process

* Increased citations of published articles through promotions

* Reduced timeline for article publication

Submit your articles and experience a new surge in publication services (https://www.peertechz.com/submission).

Peertechz journals wishes everlasting success in your every endeavours.

Copyright: @ 2020 Al-Maawali M, et al. This is an open-access article distributed under the terms of the Creative Commons Attribution License, which permits unrestricted use, distribution, and reproduction in any medium, provided the original author and source are credited.

Citation: Al-Maawali M, Al Rawahi S, Al-Qadhi H (2020) Trauma Resuscitation Time at A Tertiary Care Hospital in Oman: A Retrospective Observational Study. Open J Trauma 4(1): 028-031. DOI: https://dx.doi.org/10.17352/ojt.000028 